\title{
Cross-Hemispheric Collaboration and Segregation Associated with Task Difficulty as Revealed by Structural and Functional Connectivity
}

\author{
(1) Simon W. Davis and Roberto Cabeza \\ Center for Cognitive Neuroscience, Duke University, Durham, North Carolina 27708
}

\begin{abstract}
Although it is known that brain regions in one hemisphere may interact very closely with their corresponding contralateral regions (collaboration) or operate relatively independent of them (segregation), the specific brain regions (where) and conditions (how) associated with collaboration or segregation are largely unknown. We investigated these issues using a split field-matching task in which participants matched the meaning of words or the visual features of faces presented to the same (unilateral) or to different (bilateral) visual fields. Matching difficulty was manipulated by varying the semantic similarity of words or the visual similarity of faces. We assessed the white matter using the fractional anisotropy (FA) measure provided by diffusion tensor imaging (DTI) and crosshemispheric communication in terms of fMRI-based connectivity between homotopic pairs of cortical regions. For both perceptual and semantic matching, bilateral trials became faster than unilateral trials as difficulty increased (bilateral processing advantage, BPA). The study yielded three novel findings. First, whereas FA in anterior corpus callosum (genu) correlated with word-matching BPA, FA in posterior corpus callosum (splenium-occipital) correlated with face-matching BPA. Second, as matching difficulty intensified, crosshemispheric functional connectivity (CFC) increased in domain-general frontopolar cortex (for both word and face matching) but decreased in domain-specific ventral temporal lobe regions (temporal pole for word matching and fusiform gyrus for face matching). Last, a mediation analysis linking DTI and fMRI data showed that CFC mediated the effect of callosal FA on BPA. These findings clarify the mechanisms by which the hemispheres interact to perform complex cognitive tasks.
\end{abstract}

Key words: connectivity; diffusion; face processing; interhemispheric; semantic memory; white matter

\section{Introduction}

Understanding cross-hemispheric communication is one of the most fundamental goals in brain science, yet this form of information transmission remains a fundamentally under-explored phenomena. Several lines of research suggest that sometimes brain regions in one hemisphere operate in direct communication with homologous regions in the contralateral hemisphere (collaboration), whereas other times, they function relatively independent of their contralateral counterparts (segregation; van der Knaap and van der Ham, 2011). Yet, it is still uncertain where (i.e., what brain regions) and how (i.e., what conditions) these patterns of collaboration or segregation emerge.

We investigated these issues using a split field-matching task-a task in which participants match a pair of stimuli (e.g., words, faces) flashed to the same or opposite visual fields (unilat-

Received Jan. 31, 2015; revised April 22, 2015; accepted April 23, 2015.

Author contributions:S.W.D. and R.C. designed research; S.W.D. performed research; S.W.D. contributed unpublished reagents/analytic tools; S.W.D. analyzed data; S.W.D. and R.C. wrote the paper.

This work was supported by National Institutes of Health Grant R01 AG19731 to R.C. S.W.D. was supported by National Institute on Aging Grant F31 AG032799.

The authors declare no competing financial interests.

Correspondence should be addressed to Simon W. Davis, Center for Cognitive Neuroscience, Duke University, Box 90999, Durham, NC 27701. E-mail: simon.davis@duke.edu.

DOI:10.1523/JNEUROSCI.0464-15.2015

Copyright $\odot 2015$ the authors $\quad 0270-6474 / 15 / 358191-10 \$ 15.00 / 0$ eral vs bilateral). Regarding the where question, we investigated two domains likely known to involve different brain networks: semantic word-matching task and visual face matching. Regarding the how question, we investigated the conditions that modulate collaboration versus segregation. We focused on difficulty, because it is well established that in this paradigm bilateral trials become faster than unilateral trials as difficulty increases (for review, see Banich and Belger, 1990), an effect known as bilateral processing advantage (BPA). Critically, we linked the BPA to MRI measures of structural and functional connectivity. We specifically investigated three questions.

First, how is cross-hemispheric communication for different domains affected by structural connectivity? We have previously shown that word-matching BPA correlates with the white-matter measure fractional anisotropy (FA) in the anterior corpus callosum (genu; Davis et al., 2012). Given that visual processing has been linked to the posterior corpus callosum (splenium-occipital; Schulte et al., 2010), we expected that genu FA would predict word-matching BPA and that FA in the splenium-occipital would predict face-matching BPA.

Second, how is cross-hemispheric communication in domaingeneral versus domain-specific regions affected by processing demands? We define domain-general regions as areas that contribute to many different domains (e.g., anterior frontal cortex), whereas domain-specific regions contribute primarily to one domain, 
such as the processing of words or processing of faces (e.g., anterior temporal or fusiform cortex, respectively). While a number of studies have established the effects of processing demands on hemispheric dominance (for review, see Ivry and Robertson, 1998), the focus of the current analysis is connectivity between the hemispheres, and not the relative differences in activity in left and right hemispheres. Thus, to answer this question we measured functional connectivity between homotopic pairs of cortical regions (cross-hemispheric functional connectivity, CFC) while subjects performed two tasks with parametric variations in task difficulty. Previous evidence suggests that as difficulty increases, CFC tends to increase in domain-general regions, such as anterior PFC (Kitzbichler et al., 2011), while decreases in CFC related to stimulus type have been previously found in domainspecific regions (Seghier et al., 2011). This suggests a regional heterogeneity in how a cortical region may engage with its crosshemisphere homolog during difficult cognitive tasks. We therefore focused on the effects of domain and difficulty on cross-hemispheric communication and predicted that, as matching difficulty increases, both word- and face-matching CFC would increase in domaingeneral regions, whereas CFC would decrease in domain-specific regions.

Finally, what is the relationship between the structural and functional mechanisms of cross-hemispheric communication? Electrophysiological studies in animals have shown that crosshemispheric interactions between homologous regions of visual cortex depend on the integrity of white-matter fibers passing through the posterior callosum (Berardi et al., 1987). In humans, CFC measures are closely related, but not isometric, with diffusion tensor imaging (DTI) measures of corpus callosum (Honey et al., 2009; Betzel et al., 2014). In the current study, we predicted that CFC between cortical regions connected by anterior callosal fibers would mediate the effect of genu FA on word-matching BPA, whereas CFC between regions linked by posterior callosal fibers would mediate the effect of splenium FA on face-matching BPA.

\section{Materials and Methods}

Participants. Seventeen younger adults were paid for their participation in this study. All participants were healthy, right-handed native English speakers with no history of neurological or psychiatric episodes (nine females; mean age: $21.7 \pm 2.6$; education: $15.1 \pm 1.7$ years; Edinburgh Handedness Scale: $96.5 \pm 8.7$ ), and had normal or corrected vision. Written informed consent was obtained from each participant, and the study met all criteria for approval from the Duke University Institutional Review Board.

Stimuli. While some studies investigating higher order visual processing have found that cerebral dominance becomes pronounced with bilateral compared with unilateral visual hemifield presentation (Han et al., 2002), these hemispheric dominance interactions may also be described within the context of cross-hemispheric cerebral connectivity. Thus, the focus of the present analysis was connectivity between the hemispheres, and not the relative differences in activity in left and right hemispheres. Nonetheless, we sought to balance the degree to which the left or right hemisphere should be recruited by our task. Thus, two types of stimuli were used in the split field-matching task — words and faceswhich have been shown to be generally left and right lateralized, respectively (Ivry and Robertson, 1998). The words were nouns selected from the University of South Florida word-association norms (Nelson et al., 2004). We chose word pairs that did not differ in their forwardassociation or backward-association strength, and used the mean association strength as a measure of symmetrical semantic distance, which was evenly distributed from high (1.0) to low (0.01) values. We selected a trimodal distribution of semantic distance values to generate word lists for easy $(0.8-1.0)$, medium $(0.4-0.6)$, and hard (0.01-0.2) difficulty
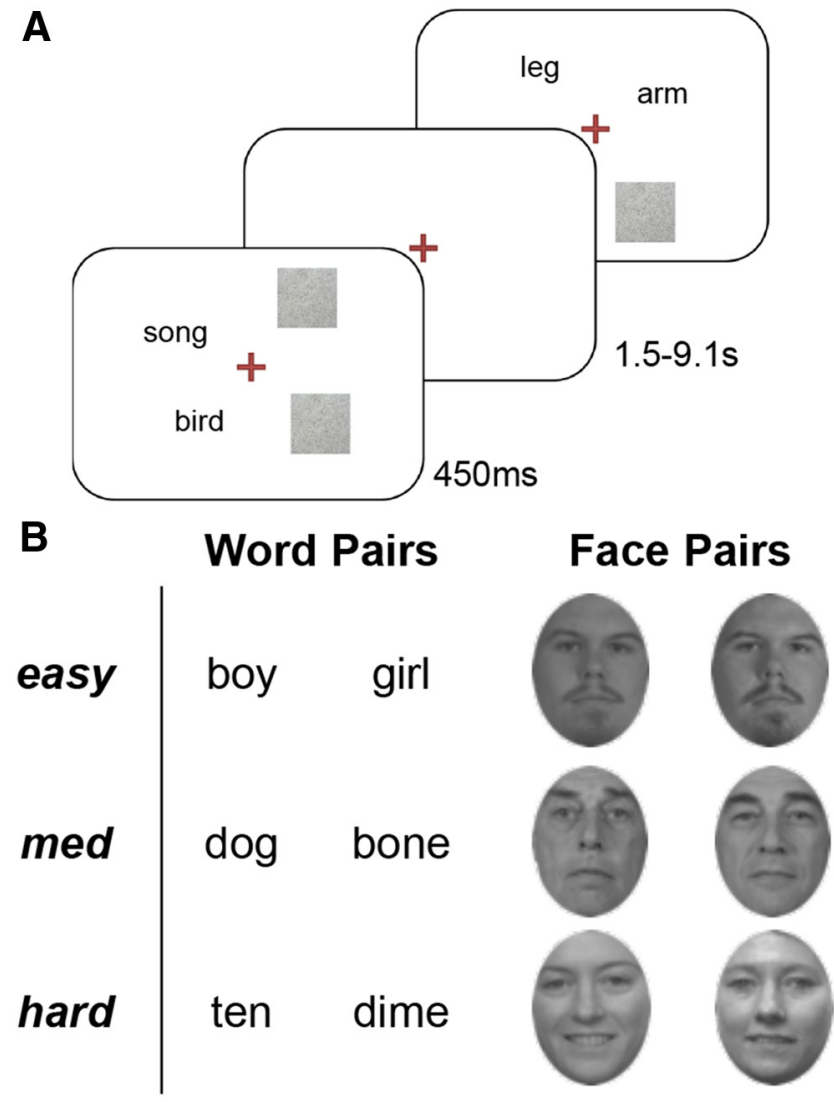

Figure 1. Behavioral task. Stimulus design and presentation for word and face matching. In each task subjects were presented with an item pair for $450 \mathrm{~ms}$ and asked to decide if the two items presented were related (either semantically or familially); an example from the wordmatching task is presented in $\boldsymbol{A}$. Intertrial response intervals varied from 1.5 to $9.1 \mathrm{~s}$. $\boldsymbol{B}$, Similarity between items was categorized into three categories; word similarity was defined by symmetrical semantic distance (Nelson et al., 2004), whereas face pair similarity was adjusted by morphing.

conditions. The word lists in each condition were matched in frequency, abstractness, and imageability (nonetheless, the relationship between words became necessarily more abstract with increasing difficulty). Face stimuli were selected from the FERET database (Phillips et al., 1998); face pairs were paired according to gender, age group (young, middle-aged, and older adults), and race. Face A of a pair was morphed with Face B using Abrosoft FantaMorph by selecting over 40 face landmarks per pair and using morphs of $20 / 40 / 60 \%$ to create easy/medium/hard difficulty matching trials, respectively (Fig. 1).

Split field-matching tasks. Participants performed three runs of a semantic word-matching task (henceforth: word task) and three runs of visual face-matching task (henceforth: face task). In both tasks, subjects were asked to fixate for the duration of the trial period, while a pair of stimuli was presented for $450 \mathrm{~ms}$ in each trial, and followed by a jittered intertrial interval from 1.5 to $9.1 \mathrm{~s}$. The stimuli were projected onto a screen $1.1 \mathrm{~m}$ away, which participants viewed through an angled mirror. In the word task, two words (e.g., ten dime) were presented and participants were asked to respond as to whether the two items were semantically related, whereas in the face task, two faces were presented and they indicated whether they were familially related (as the faces of relatives) or dissimilar. Participants were instructed to respond as fast and accurately as possible by pressing one of two keys placed under first and second fingers. There were 360 matching trials that required a "similar" response (180 semantically related words and 180 morphed faces) intermixed with 60 nonmatching that required a "dissimilar" response (30 unrelated words and 30 unmorphed faces). The nonmatching trials were included to ensure task performance ("catch trials") but were not included in fMRI analyses. 
In both word and face tasks, there were unilateral and bilateral presentation conditions. In the unilateral condition, two words or faces were presented in the same visual field (i.e., both in the left or both in the right) whereas in the bilateral condition, they were presented in opposite visual fields (e.g., one in the left and the other in the right). The distance between the two items, and from fixation, was between 2.1 and $4.1^{\circ}$. Participants were instructed to maintain fixation on a cross in the center of the screen at all times. Eye movements were monitored with a stereoscopic eye tracker (Resonance Technologies), which recorded pupil position once every $10 \mathrm{~ms}$. Trials with mean lateral eye movements $>5$ degrees over a $50 \mathrm{~ms}$ time window were excluded from the analyses.

Behavioral analysis. We first tested whether the unilateral condition exhibited any laterality effects, and found no differences in response time or accuracy ratings between left- and right-lateralized presentations in any of the three difficulty conditions. For both word and face tasks, we performed separate 3 (Difficulty: easy, medium, hard) $\times 2$ (Field: unilateral, bilateral) repeated-measures ANOVAs on response times (RTs) and accuracy rates (four ANOVAs in total). In all ANOVA models described, assumptions of sphericity (i.e., equal variance among Difficulty and Field condition factors) were not violated (all ps $>0.05$ ). Furthermore, because a handful of subjects performed at ceiling at the lowest levels of Difficulty, we used only correct trials in the RT ANOVAs. Within-subject contrasts for both linear and quadratic effects of Difficulty were performed to more fully describe the pattern of differences across stimulus types at varying levels of task demand. We expected linear increases for both the difficulty main effect and the Difficulty $\times$ Field interaction. Last, consistent with previous literature (Schulte et al., 2010), we computed the BPA measure based on correct trials, in which unilateral-bilateral RT differences were weighted by Difficulty level (easy, medium, and hard):

$$
\begin{array}{r}
B P A=\left[\left(\left(U_{\text {easy }}-B_{\text {easy }}\right) * 1\right)+\left(\left(U_{\text {medium }}-B_{\text {medium }}\right) * 2\right)\right. \\
\left.\left.+\left(\left(U_{\text {hard }}-B_{\text {hard }}\right) * 3\right) / 3\right)\right] .
\end{array}
$$

Consistent with the idea that bilateral interactions should increase linearly with increasing effort (Kitzbichler et al., 2011), we weighed our discrete difficulty levels (easy, medium, and hard) linearly; the use of such a weighting allows us to both incorporate a simple and realistic hypothesis about the linear nature of BPA-related increases and reduce the number of statistical tests of the data.

Lateralized choice RT task. Before scanning, participants performed a simple lateralized choice RT task that is frequently used in behavioral index of the speed of cross-hemispheric communication (Poffenberger, 1912). This behavioral paradigm (often known as the crossed-uncrossed difference or CUD task) provides a behavioral index of interhemispheric transfer time (Schulte et al., 2010; Linnet and Roser, 2012), and therefore faster scores suggest greater efficiency between the hemispheres. If the BPA emerges from a fast, efficient relay between the hemispheres, it is logical to expect that lower interhemispheric transfer times would promote this behavioral effect; therefore, we hypothesized that subjects with lower CUD scores would show the largest BPAs. In each trial, a black circle on a white background (size: $2-4^{\circ}$ ) was presented for $50 \mathrm{~ms}$ (stimulus onset asynchrony: $2 \mathrm{~s}$ ) in the left or the right visual field ( $8^{\circ}$ from vertical meridian), and participants pressed a key as soon as they detected the stimulus, either with the hand on the same side of the stimulus (uncrossed condition) or with the opposite hand (crossed condition). For 100 trials, they pressed the key with the index finger of one hand and for 100 trials with the index finger of the other hand, with hand order counterbalanced across subjects. Responses to uncrossed trials are usually faster than crossed trials; hence, the CUD in RTs is typically positive ( $\sim 5-10 \mathrm{~ms}$ in healthy young adults). Effectively, we used the CUD to investigate if participants showing faster cross-hemispheric communication show a larger BPA.

MRI scanning. Participants were scanned on a 3 T gradient-echo scanner (General Electric 3.0 tesla Signa Excite HD short-bore scanner, equipped with an 8-channel head coil). Coplanar functional images were acquired using an inverse spiral sequence $(64 \times 64$ matrix, $\mathrm{TR}=1700$ $\mathrm{ms} ; \mathrm{TE}=31 \mathrm{~ms}$, FOV $=240 \mathrm{~mm}$, 37 slices, $3.8 \mathrm{~mm}$ slice thickness, 254 images). The DT-MRI dataset was based on a single-shot EPI sequence $(\mathrm{TR}=1700 \mathrm{~ms}, 50$ contiguous slices of $2.0 \mathrm{~mm}$ thickness, $\mathrm{FOV}=256 \times$ $256 \mathrm{~mm}^{2}$, matrix size $128 \times 128$, voxel size $2 \times 2 \times 2 \mathrm{~mm}$, b-value $=$ $1000 \mathrm{~s} / \mathrm{mm}^{2}, 15$ diffusion-sensitizing directions, 960 total images, total scan time $\sim 5 \mathrm{~min})$. The anatomical MRI was acquired using a 3D T1weighted echo-planar sequence $(256 \times 256$ matrix, $\mathrm{TR}=12 \mathrm{~ms}, \mathrm{TE}=5$ $\mathrm{ms}, \mathrm{FOV}=24 \mathrm{~cm}$, 68 slices, $1.9 \mathrm{~mm}$ slice thickness, 248 images). Scanner noise was reduced with earplugs and head motion was minimized with foam pads. Behavioral responses were recorded with a four-key fiber optic response box (Resonance Technology), and when necessary, vision was corrected using MRI-compatible lenses that matched the distance prescription used by the participant.

FA of the corpus callosum. FA is a measure provided by the analysis of diffusion-weighted images. These images were skull stripped to remove nonbrain and ambient noise using the brain extraction tool (Smith et al., 2002), and eddy corrected to account for drifts in scanner acquisition. Diffusion tensors were calculated from the 15 diffusion weighted images based on a typical, simple least-squares fit of the tensor model to the diffusion data, using the freely available software medINRIA (http://med.inria.fr/; Toussaint et al., 2007). Fiber-tracking parameters included a minimum FA of 0.2 and an angle of maximum deviation of $35^{\circ}$. The tractography algorithm was based on trilinear log-Euclidean interpolation and used a standard streamline algorithm (Xu et al., 2002); fiber tracking was automatic, since every voxel of the reference volume was a seeding point (Fillard et al., 2007). The present analysis focused on interhemispheric connectivity and thus we restricted our analysis to the corpus callosum, which connects homologous regions of all parts of the cortex (Aboitiz et al., 1992), divided into six regions of interest and guided by callosal anatomical projections described by Pandya and Seltzer (1986); a similar division among callosal divisions using diffusion tractography is described previously (Sullivan et al., 2006). We first limited subjects' full-brain tractography results to callosal fibers traversing the midline, ignoring noncallosal pathways such as the anterior and posterior commissures, or the hippocampal commissure. We then segregated tract streamlines into six groups by manually tracing a set of ROIs describing sections of the cross-sectional corpus callosum (i.e., genu, anterior body, body, tapetum, splenium-parietal, and spleniumoccipital sections; Fig. 3A) based on canonical divisions of the midline corpus callosum (for typical sectional volumes, see Pandya and Seltzer, 1986), and limiting each sectional fiber population to those passing through a given ROI. We then "cut" all callosal pathways $5 \mathrm{~mm}$ lateral (plus or minus) to the midline, and FA values (based on the initial tensor calculation described above) from the resulting fiber populations were averaged within a callosal division. We focused on FA values, as the number of tract streamlines within a tract group may be more susceptible to study-specific variations in image acquisition, voxel size, or tractography algorithm (Jones et al., 2013). All callosal ROIs and fiber tracts remained in native space throughout the fiber-tracking procedure. Reliability of user-created diffusion tractography data was assessed using methods previously described by our lab (Davis et al., 2009) and previously (Wakana et al., 2007; Madden et al., 2009). Briefly, the volume of all callosal ROIs and tracts were evaluated for the voxel extent of overlap within and between expert raters, and were accepted as long as intrarater and inter-rater reliability ratings exceeded a "high" rate of reliability (Cohen's $\kappa>0.8$ ), according to criteria set forth by Landis and Koch (1977).

fMRI analyses. Preprocessing and data analysis were performed using SPM8 (Wellcome Department of Cognitive Neurology, London, UK) and custom MATLAB scripts. After discarding the six initial volumes to allow for scanner stabilization, images were slice-time and motion corrected, spatially normalized to the MNI template, and smoothed using an $8 \times 8 \times 8 \mathrm{~mm}$ Gaussian kernel. Event-related BOLD responses for correct trials were analyzed using a modified general linear model (Worsley and Friston, 1995). Because the word matching should depend primarily on left hemisphere processing and faces primarily on the right hemisphere, and the main goal was to examine recruitment beyond this initial task-specific activation and independent of well known hemispheric dominance biases, we excluded from the analyses trials on which the words were presented unilaterally to the right hemisphere, or faces pre- 
sented to the left hemisphere. Furthermore, because of the neural evidence for $2-3^{\circ}$ of bilateral representation of the parafovea (Lavidor and Walsh, 2004), stimulus presentation in the unilateral condition likely involved some degree of bilateral representation in early visual cortices. Nonetheless, such a perfect unilateral condition is not critical for the main conclusions of the current analysis, which are related to the effects of difficulty for different types of stimuli and the relationship between functional and structural connectivity. Catch trials (nonmatches), incorrect trials, no-response trials, and trials with more than one response were modeled explicitly as nuisance regressors and not considered in the main analysis.

CFC analyses. Our CFC analyses proceeded on the hypothesis that domain-specific regions for semantic word and visual face processing should show fundamentally different CFC patterns than domain-general regions. The analyses consisted of three main steps. First, we defined stimulus-specific versus stimulus-independent ROIs and control ROIs. To avoid circularity in the definition of domain-specific and domainindependent ROIs, we performed a meta-analysis on the fMRI literature to identify brain regions associated with words, faces, and domain-general processes. Using the Neurosyth activation database (http://neurosynth.org; Yarkoni et al., 2011), we extracted reverse inference maps of regions associated with general cognitive demands (keywords = "associative" + "task-positive" + "higher-order"; 282 studies), or associated specifically with semantic word processing (keywords $=$ "words" + "semantic"; 634 studies) or visual face processing (keywords = "faces"; 577 studies). Then, we thresholded the maps at $p<$ $0.001(z=3.56)$, and peak coordinates from above-threshold clusters were used to select ROIs from the Harvard Oxford Atlas (HOA; Desikan et al., 2006). For both left- and right-sided peaks identified by the Neurosynth maps, we considered both the left ROI and its contralateral homolog in the right; the Neurosynth coordinates and the HOA ROI labels are shown in the leftmost columns of Table 3.

Second, functional connectivity between contralateral ROIs was calculated using the $\beta$-correlations method (Rissman et al., 2004), which has been applied to neuroimaging designs with similar timing parameters (Daselaar et al., 2006; Axmacher et al., 2008; Atlas et al., 2010), and provides a more reliable and powerful estimate of functional connectivity in event-related designs than other methods, including psychophysiological interaction analysis), which are better suited to blocked designs (Cisler et al., 2014). Specifically, we first created a GLM in which each trial was modeled as a separate covariate and yielded parameter estimates (betas) for each individual trial and participant. These first-level models produce $\beta$-coefficients corresponding to the ability of each HRFconvolved trial regressor in predicting the fMRI time series; these $\beta$-values were then concatenated for each trial type and for each ROI in one hemisphere were correlated (Pearson's $r$ ) with the corresponding contralateral ROI within each subject. The resulting correlations, representing the CFC between each pair of cortical ROIs, were Fishertransformed to adjust for individual subject variation in correlational range.

Last, to investigate the hypothesis that the relationship between CFC and BPA is partially due to differences in white-matter quality assessed as FA we performed a mediation analysis using these three measures (Baron and Kenny, 1986). A mediation analysis can be conceptualized as three separate regression analyses testing each component of the mediation hypothesis as follows: (1) the relationship between the callosal FA and CFC (the $a$ effect; Fig. 3); (2) the relationship between CFC and the BPA, controlling for callosal FA (the $b$ effect); and (3) the extent to which the inclusion of a mediating variable (CFC) attenuates the observed effects of the predictor (FA) on the outcome variable (BPA) covariance after the inclusion of the mediator (the $a b$ effect, based on the difference between the total [unmediated] path $c$, and the direct path $c^{\prime}$ between predictor and outcome variable). Thus, the logic of the applied mediation model is that the structure-behavior relationships identified above can be at least partially explained by the degree to which individuals coactivate contralateral regions of cortex.
Table 1. Demographics results

\begin{tabular}{lc}
\hline Demographics & Mean (STD) \\
\hline Age & $21.70(2.6)$ \\
Education (years) & $15.06(1.7)$ \\
Edinburgh Handedness Scale & $96.5(8.7)$ \\
Crossed-uncrossed difference (ms) & $4.5(0.42)$ \\
\hline
\end{tabular}

\section{Table 2. Behavioral results}

\begin{tabular}{lcll}
\hline & Easy & Medium & Hard \\
\hline $\begin{array}{l}\text { Word-matching task (scanned) } \\
\text { Accuracy (proportion correct) }\end{array}$ & & & \\
$\quad$ Unilateral & $0.91(0.10)$ & $0.82(012)$ & $0.73(0.15)$ \\
$\quad$ Bilateral & $0.90(0.10)$ & $0.85(0.13)$ & $0.75(0.16)$ \\
$\quad$ Reaction time (ms) & $1241(188)$ & $1399(231)$ & $1560(270)$ \\
$\quad$ Unilateral & $1198(189)$ & $1349(272)$ & $1420(264)$ \\
$\quad$ Bilateral & $92.18(27.4)$ & & \\
$\quad$ Bilateral processing advantage (ms) & & & \\
Face-matching task (scanned) & & & \\
$\quad$ Accuracy (proportion correct) & $0.84(0.15)$ & $0.75(0.14)$ & $0.56(0.20)$ \\
$\quad$ Unilateral & $0.86(0.13)$ & $0.77(0.14)$ & $0.62(0.18)$ \\
$\quad$ Bilateral & & & \\
$\quad$ Reaction time (ms) & $1114(220)$ & $1289(267)$ & $1340(301)$ \\
$\quad$ Unilateral & $1117(185)$ & $1201(210)$ & $1322(275)$ \\
$\quad$ Bilateral & $38.33(21.97)$ & & \\
Bilateral processing advantage (ms) & & & \\
\hline
\end{tabular}

\section{Results}

\section{Behavioral data}

\section{Split field-matching task}

The main results of the split field-matching task are RTs, because differences in accuracy rates are usually not significant (Weissman et al., 2000; Butcher and Cavanagh, 2008). Less than $10 \%$ of trials were removed in each subject due to lateral eye movements. Tables 1, 2 and Figure 2 summarize accuracy and RTs in the word and face matching. As expected, the ANOVA on RTs during word matching yielded a main effect of Difficulty $\left(F_{(1,16)}=22.15, p<\right.$ $0.001)$ and a main effect of Field condition $\left(F_{(1,16)}=30.13, p<\right.$ $0.001)$. Critically, consistent with the split field-matching literature (Banich, 1998), there was a significant Difficulty $\times$ Field condition interaction $\left(F_{(1,16)}=4.99, p=0.04\right)$ because the Bilateral condition was faster than the Unilateral condition as difficulty increased (BPA). Within-subject contrasts indicated that both the main effect of Difficulty $\left(F_{(1,16)}=32.32, p<0.001\right)$ and the Difficulty $\times$ Field interaction $\left(F_{(1,16)}=6.72, p=0.02\right)$ were linear rather than quadratic, indicating that BPA increases gradually with Difficulty. The ANOVA on RTs during face matching yielded a main effect of task Difficulty $\left(F_{(1,16)}=12.26, p=0.003\right)$, a nonsignificant main effect of Field $\left(F_{(1,16)}=0.83, p=\mathrm{NS}\right)$ and, critically, a significant Difficulty $\times$ Field interaction $\left(F_{(1,16)}=\right.$ $4.57, p=0.048)$. The main effect of Difficulty was linear $\left(F_{(1,16)}=\right.$ 20.83, $p<0.001)$ but not quadratic, whereas the Difficulty $\times$ Field interaction was quadratic $\left(F_{(1,16)}=4.76, p<0.044\right)$. As illustrated by Figure $2 B$, this quadratic effect occurred because the BPA increased from easy to medium conditions but then decreased in the hard condition.

Turning to accuracy rates, in keeping with the literature, differences were mostly nonsignificant. Word matching showed significant Difficulty $\left(F_{(1,16)}=5.12, p=0.037\right)$ and Difficulty $\times$ Field interaction $\left(F_{(1,16)}=5.42, p=0.03\right)$ effects but the main effect of Field $\left(F_{(1,16)}=0.07, p=\right.$ NS) did not. Face matching showed a main effect of Difficulty $\left(F_{(1,16)}=5.07, p=0.039\right)$ but neither the main effect of Field nor the Difficulty $\times$ Field inter- 

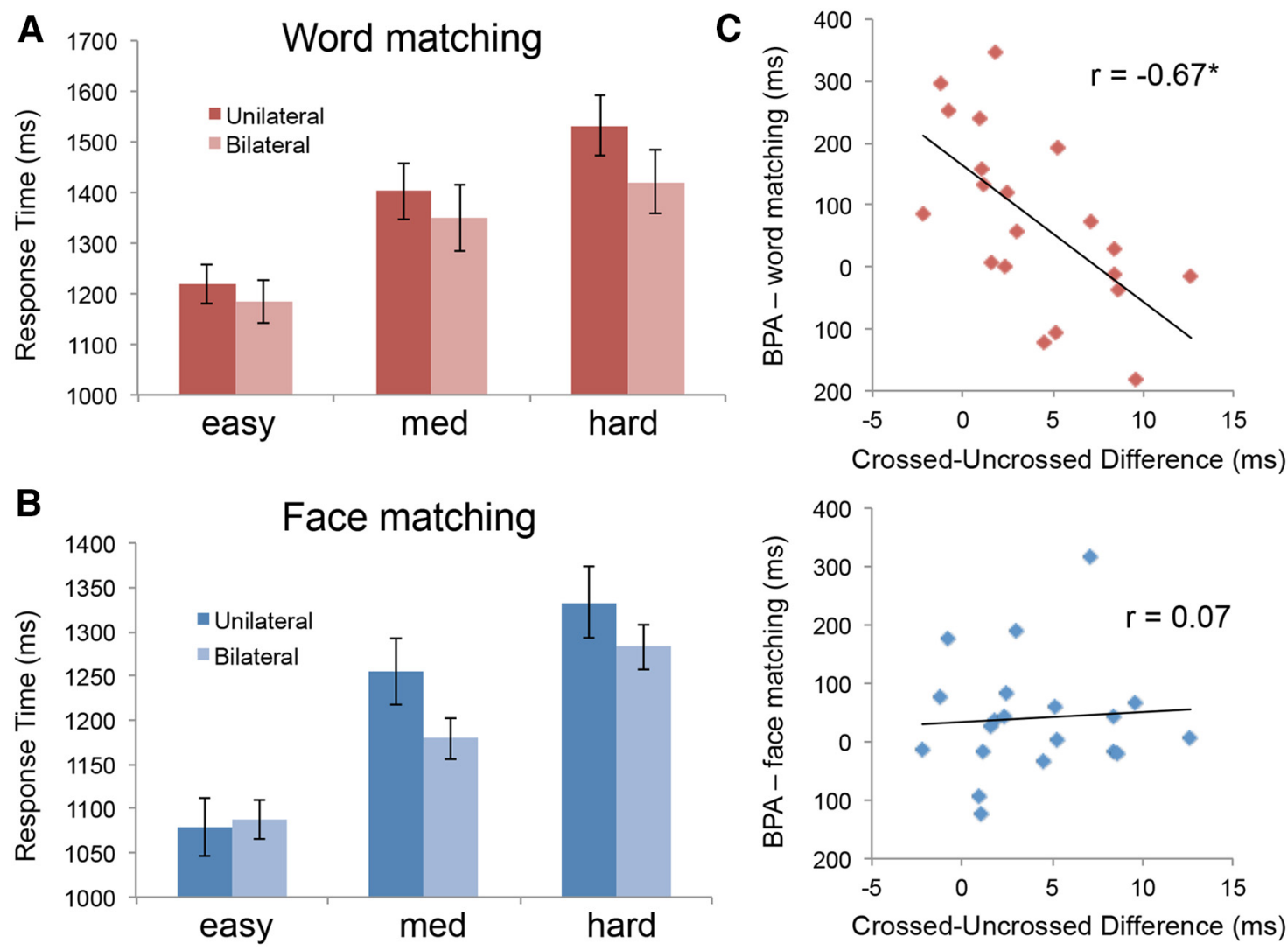

Figure 2. Behavioral results. Response times in word matching $(\boldsymbol{A})$ and face matching $(\boldsymbol{B})$ both show a clear increase in response times with increasing Difficulty; however, differences between Unilateral and Bilateral presentations became evident at greater levels of Difficulty. $C$, Our derived measure of the speed advantage for BPAs in the word matching showed an inverse relationship with the CUD; the perceptual BPA did not demonstrate a similar relationship.

actions were significant. Therefore, for the rest of this manuscript we focus on RT effects, which showed consistently with the literature that showed significant BPA for both word and face matching and significant difficulty effects.

\section{Lateralized choice RT task}

As mentioned in Materials and Methods, before scanning, participants performed a lateralized choice RT task in which they responded to stimuli presented to the left or right visual fields using the hand on the same side or on the opposite side. Although the cross-uncrossed RT difference (mean: $4.5 \pm 0.42 \mathrm{~ms}$ ) was not reliably correlated with the BPA in face matching, it was significantly correlated with the BPA for word matching (Fig. $\left.2 C ; r_{(16)}=-0.67, p=0.002\right)$. This intuitive result suggests that subjects with lower cross-hemispheric transfer times showed a greater benefit of bilateral presentations (larger BPA). This finding is consistent with the hypothesis that BPA does not occur for easy tasks due to the initial cost in crosshemispheric transfer because reduced transfer time would also reduce this cost.

\section{Structural connectivity and the BPA}

Our first question was about the effects of domain (word matching vs face matching) on the white-matter mechanisms of crosshemispheric communication, which we measured using the FA measure yielded by DTI analyses. Mean FA values within callosal sections (genu: 0.60; anterior body: 0.63; body: 0.65; tapetum: 0.73 , splenium-parietal: 0.72 ; splenium-occipital: 0.74 ; all SD $<$ 0.05 ) were consistent with previously published reports (Sullivan et al., 2006), and reflect the general anterior-posterior differences in callosal fiber composition. We then calculated Pearson's $r$ correlations between BPA and FA separately for word and face matching and for each of the six callosal regions (genu, anterior body, body, tapetum, splenium-parietal, and splenium-occipital). As illustrated by Figure 3, these analyses confirmed our first prediction that word-matching BPA correlates with FA in the genu $\left(r_{(16)}=0.58, p=0.01\right)$, whereas face-matching BPA correlates with occipital splenium FA. This last effect was found specifically for splenium-occipital fibers $\left(r_{(16)}=0.58, p=0.01\right)$, whereas splenium-parietal FA actually showed correlation with word-matching BPA $\left(r_{(16)}=0.67, p=0.002\right)$. The observed relationships remained significant after controlling for both sex and total brain volume.

\section{Cross-hemispheric functional connectivity}

Our second question concerned the effects of domain (word matching vs face matching) on the functional correlates of crosshemispheric communication, which we assessed in terms of functional connectivity between corresponding contralateral regions (CFC). In particular, we focused on our hypothesis that domain-general regions respond to difficulty by collaborating and domain-specific regions by segregating. To investigate this hypothesis, we defined domain-general and domain-specific ROIs independently of our data using Neurosynth (see Materials and Methods). We collapsed difficulty conditions across bilateral and unilateral presentations to reduce the number of statistical comparisons. The two rightmost columns of Table 3 indicate whether the effect of difficulty on CFC during word and face matching was positive or negative, with bold numbers indicating 
significant effects. Clearly, difficulty effects on CFC were mostly positive in domain-general ROIs (greater difficulty and increased CFC), whereas the opposite was true in domain-specific ROIs (greater difficulty and reduced CFC), consistent with our prediction. As illustrated by Figure 4, as matching difficulty intensifies, $\mathrm{CFC}$ increases for both word and face matching in a domain-general region anterior PFC, but it decreases in domainspecific regions. As shown by the line plots, whereas in regions associated with semantic processing of words (e.g., temporal pole and ventrolateral PFC) CFC decreases with difficulty for word matching but not for face matching, in regions associated with face processing, CFC decreases with difficulty for face matching but not for word matching. No effects of difficulty on CFC were observed in control regions (primary motor and primary visual cortices). The dissociation between domain-general and domain-specific regions supports our hypothesis that as difficulty increases, domain-general regions tend to collaborate and domain-specific regions tend to segregate.

\section{Linking FA and CFC results: mediation analysis}

Our final question was about the relationship between white matter and functional correlates of cross-hemispheric communication. We hypothesized that BPA depends on CFC, which in turns depends on the corpus callosum. To investigate this hypothesis we performed mediation analyses across all sections of the corpus callosum that demonstrated a relationship between FA and BPA (i.e., genu, anterior and posterior of the splenium) and regions showing an effect of Difficulty on CFC connected by the corresponding callosal fiber systems [e.g., genu: frontal pole, temporal pole, inferior frontal gyrus-pars triangularis (IFG-PT); splenium-occipital: fusiform cortex]. We observed a regionally specific pattern of mediation (Fig. 5), such that for word matching, CFC between regions connected by genu fibers, including the frontal pole, accounted for a portion of the covariance between genu FA and the word-matching BPA ( $b$ effect: $t_{(16)}=2.78, p=0.013 ; a b$ effect: $t_{(16)}=3.03, p=0.008 ; c$ effect $=2.58, p=0.01, c^{\prime}$ effect $\left.=1.96, p=0.049\right)$. Conversely, during face matching, CFC between ventral occipitotemporal regions connected by occipital splenium fibers (Clarke and Miklossy, 1990; Park et al., 2008) accounted for the covariance between splenium-occipital FA and the face-matching BPA $(b$ effect: $t_{(16)}=2.62, p=0.019 ; a b$ effect: $t_{(16)}=2.37, p=0.03 ; c$ effect $=2.32, p=0.02, c^{\prime}$ effect $\left.=1.35, p=0.17\right)$. No such mediation effect was present in the genu for the face-matching task (we discuss possible interpretations of this finding below).

B
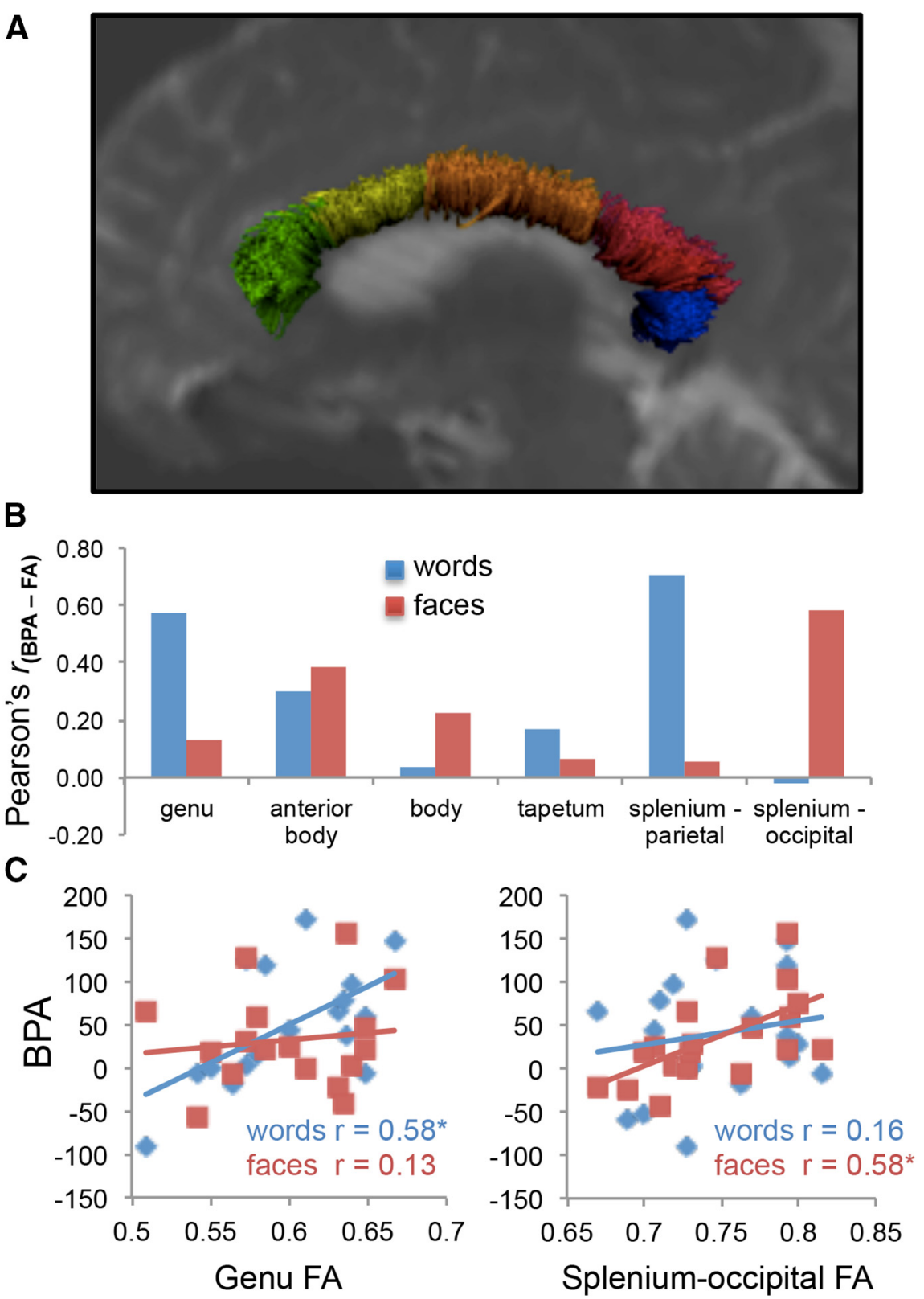

Figure 3. Effects of the callosal fractional anisotropy on the BPA. Structural connectivity supports the advantage of bilateral presentations under increasingly difficult task conditions. $\boldsymbol{A}, \mathrm{A}$ cross-sectional display of six canonical sections of the corpus callosum within a representative subject. We found that for semantic matching, the BPA is associated with greater FA in fibers passing through the genu and parietal targets through the splenium, whereas individual differences in the BPA for face matching sons). We highlight this dissociation in the anterior genu and posterior splenium-occipital fiber sections ( $\boldsymbol{C}$, demonstrating a clear regional specificity for the BPA.

CFC for IFG-PT and temporal pole also showed no mediation effects. In sum, the results were consistent with the prediction that CFC between cortical regions connected by genu fibers mediates the effect of genu FA on word-matching BPA, whereas CFC between regions linked by splenium fibers mediates the splenium FA effect on face-matching BPA.

\section{Discussion}

Although a solid understanding of the mechanisms of crosshemispheric communication is a fundamental goal in brain science, the precise conditions (how) and regions (where) associated with either increases in communication (collaboration) or 
Table 3. ROI definitions and effects of difficulty on CFC

\begin{tabular}{|c|c|c|c|c|c|c|c|c|}
\hline \multirow[b]{2}{*}{ Neurosynth map } & \multirow[b]{2}{*}{ ROls (HOA) } & \multirow[b]{2}{*}{$\mathrm{L} / \mathrm{R}$} & \multicolumn{4}{|c|}{ Neurosynth coordinates } & \multicolumn{2}{|c|}{ Difficulty effect ( $t$ ) } \\
\hline & & & $Z$ & $x$ & $y$ & $z$ & Word matching & Face matching \\
\hline \multirow[t]{5}{*}{ Domain-general } & Anterior PFC & $L / R$ & 12.89 & 3 & 56 & 30 & 3.36 & 2.98 \\
\hline & Precentral gyrus & $\mathrm{L}$ & 10.41 & -42 & 6 & 30 & 0.42 & 0.14 \\
\hline & Posterior cingulate cortex & $L / R$ & 15.38 & -2 & -52 & 30 & -0.51 & -0.29 \\
\hline & Angular gyrus & $\mathrm{L}$ & 7.91 & -52 & -58 & 35 & 1.01 & 1.08 \\
\hline & Angular gyrus & $\mathrm{R}$ & 9.15 & 50 & -62 & 28 & - & - \\
\hline \multirow[t]{4}{*}{ Word-related } & Inferior frontal gyrus, PT & $\mathrm{L}$ & 9.11 & -49 & 30 & 6 & -2.89 & -0.25 \\
\hline & Mid-frontal gyrus & $\mathrm{L}$ & 10.45 & -50 & 24 & 20 & -0.87 & 1.12 \\
\hline & Temporal pole & $\mathrm{L}$ & 5.25 & -51 & 12 & -18 & -3.14 & 0.76 \\
\hline & Inferior temporal gyrus, T0 & $\mathrm{L}$ & 13.36 & -48 & -57 & -14 & 1.24 & -0.03 \\
\hline \multirow[t]{4}{*}{ Face-related } & Amygdala & $\mathrm{R}$ & 14.18 & 24 & -4 & -22 & 1.64 & 1.03 \\
\hline & Amygdala & $\mathrm{L}$ & 14.04 & -18 & -8 & -18 & - & - \\
\hline & TO fusiform & $L$ & 16.19 & 42 & -48 & -20 & 1.19 & -3.16 \\
\hline & T0 fusiform & $L$ & 13.58 & -42 & -52 & -20 & - & - \\
\hline
\end{tabular}

L, left; PT, pars triangularis; $R$, right; T0, temporo-occipital.

\section{Domain-general}
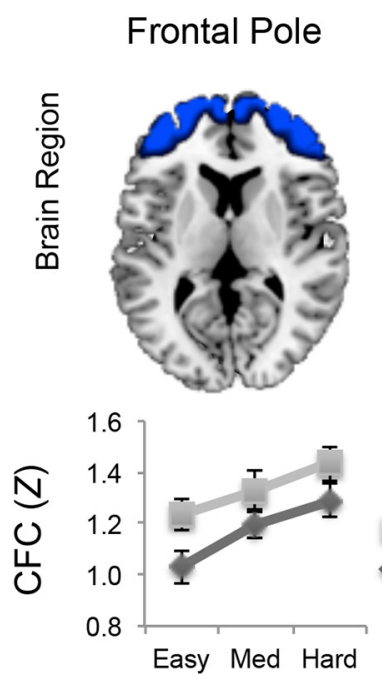

Domain-specific

Words
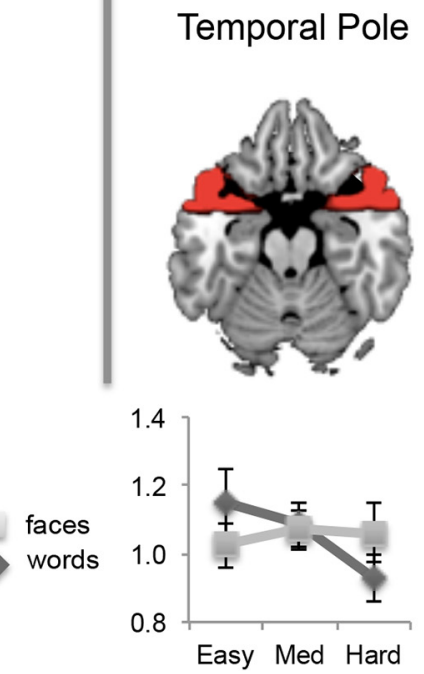

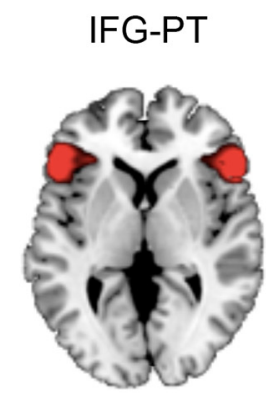

\section{Faces}

Fusiform

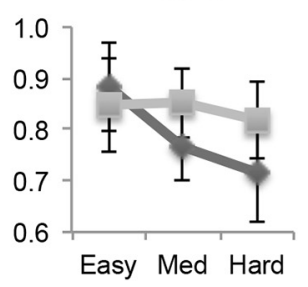

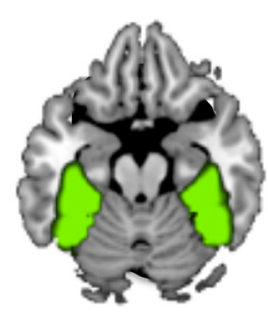

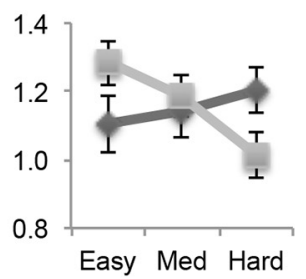

Figure 4. Domain Generality and Domain Specificity in cross-hemispheric communication. Domain-general regions defined independently exhibited domain-general increases in CFC, suggesting that these higher order bilateral systems collaborate to address the demand increase. In contrast, domain-specific regions exhibited a domain-specific decrease in CFC with increasing Difficulty. This result suggests that as processing demands increase, regions primary to the domain of processing segregate from the contralateral hemisphere.

decreases in communication (segregation) are still uncertain. To investigate these issues we linked behavioral results from the split field-matching task to MRI measures of brain white matter (DTI) and function (fMRI). The study yielded three main findings regarding the neural mechanisms of cross-hemispheric communication. First, analysis of the corpus callosum showed a double dissociation between FA in anterior and posterior callosal regions for different domains, whereas genu FA correlated with wordmatching BPA and splenium-occipital FA correlated with facematching BPA. This finding shows that the white-matter mechanisms of cross-hemispheric communication vary depending on cortical regions associated with a particular processing domain. Second, as difficulty intensified, CFC increased in the frontopolar cortex for both word and face matching, but it decreased in ventral temporal lobe regions, such as the temporal pole for word matching and the fusiform gyrus for face matching. This finding indicates that when difficulty increases domain-general regions tend to collaborate with their contralateral counterparts, whereas domain-specific regions tend to segregate from them. Last, linking structural and functional connectivity, we found that for a region connected by the genu, $\mathrm{CFC}$ mediates the effect of genu FA on word-matching BPA, whereas for regions connected by the splenium, CFC in the splenium-occipital mediates the effect of splenium FA on face-matching BPA. This finding is consistent with our hypothesis that BPA depends on CFC, which in turn depends on the corpus callosum (Davis et al., 2012). Our main three findings are discussed in separate sections below.

Linking DTI to the BPA: genu and splenium-occipital FA, respectively, predict word-matching and face-matching $\mathrm{BPA}$ Our first finding was that our DTI white-matter measure (FA) showed a clear regional specificity within the corpus callosum, such that anterior callosal fibers passing through the genu and connecting frontal regions predicted word-matching BPA, whereas posterior callosal fibers passing through the spleniumoccipital and connecting occipitotemporal regions predicted the perceptual BPA (Fig. 3). The face-matching BPA correlation with splenium FA was found in the occipital section but not in the parietal section of this bundle, which actually showed a stronger correlation with word-matching BPA, and could reflect the fact 

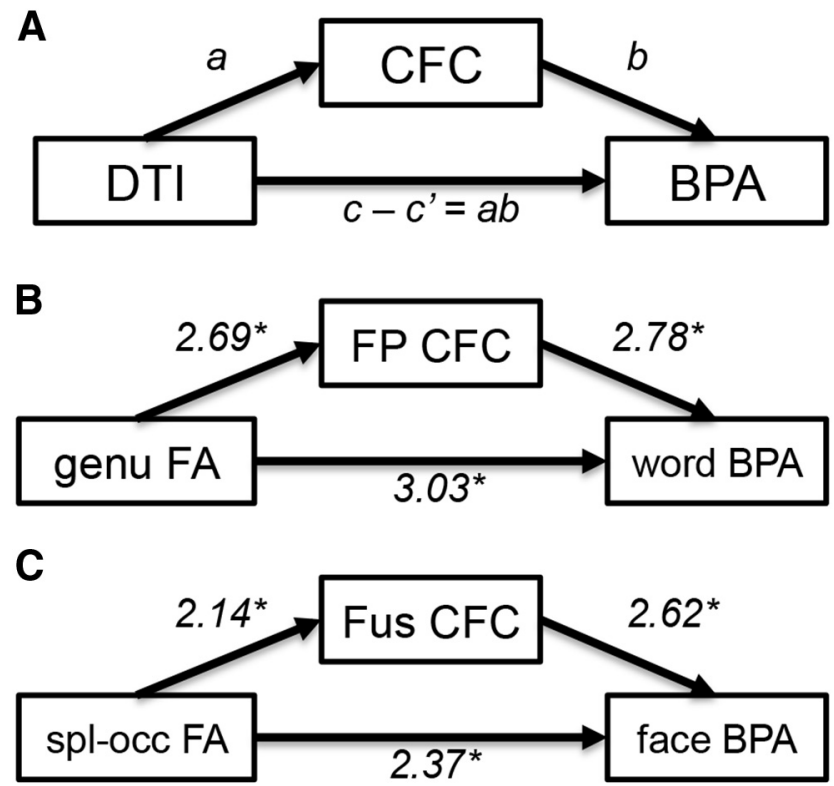

Figure 5. Mediation of the relationship between structure and behavior by functional connectivity. The conceptual logic $(\boldsymbol{A})$ posits that the observed relationship between structural connectivity (FA) and behavior (BPA; see Fig. 3 ) is at least partially explained by differences in functional connectivity (CFC). Significant mediation effects were observed in the frontopolar cortex for the word-matching task $(\boldsymbol{B})$ and posterior fusiform cortex for the face-matching task (C). Fus, fusiform; FP, frontopolar; spl-occ, splenium-occipital.

that the splenium-parietal fibers connect not only dorsal parietal regions but also ventral parietal regions associated with semantic word processing, such as the angular gyrus (Binder et al., 2009). Alternatively, the relationship of the word-matching BPA with this posterior callosal region may reflect the capacity of the left-dominant ventral visual system for words (Devereux et al., 2013) to pass inhibitory signals to the nondominant right hemisphere (see below, Effects of task difficulty on CFC: domain-general collaboration versus domain-specific segregation). Nonetheless, this account is speculative and requires further research. A critical point about our first finding is that dissociation between genu and splenium occipital regions is not merely about anatomy (genu fibers connect frontal regions and splenium-occipital fibers connect occipitotemporal regions); it is about the white matter bases of cross-hemispheric communication because FA predicted the advantage of bilateral over unilateral trials, which is a measure of collaboration in different callosal regions. To our knowledge, this is the first time this dissociation is reported in the literature.

Previous associations between the benefits of bilateral presentations and corpus callosum measures have thus far used relatively simple perceptual stimuli, including flashing geometric figures presented to one or two fields (Schulte et al., 2010; Schulte et al., 2013). Our results attempt to extend these results to two broadly different domains of complex cognitive processing, semantic word matching, and visual face matching, typically associated with relatively anterior left hemisphere regions versus relatively posterior right hemisphere regions. Thus, one answer to the where question is that semantics-related anterior regions communicate via anterior callosal fibers (genu), whereas visionrelated posterior regions communicate via posterior callosal fibers (splenium-occipital).
Effects of task difficulty on CFC: domain-general collaboration versus domain-specific segregation

Our second finding was that, as a matching difficulty intensified, CFC increased (collaboration) in the domain-general frontopolar cortex for both word and face matching, but it decreased (segregation) in the domain-specific temporopolar cortex for word matching and fusiform cortex for face matching (Fig. 4). The finding of increasing collaboration in a domain-general region effect is consistent with prior ERP and neuroimaging work, suggesting that subjects are more likely to exhibit more bilateral frontal patterns of activity when the more bilaterally associated process of meaning selection or ambiguity resolution is necessary (Rodd et al., 2005; Zempleni et al., 2007; Meyer and Federmeier, 2008). Nonetheless, the domain generality of this finding suggests parametric increases in difficulty in both word and face processing engender a beneficial role for CFC when processing demands are high; this conclusion is supported by a recent graphtheoretical analysis of MEG data showing that increases in task difficulty ( $n$-back load) increase the long-distance synchronization between contralateral brain regions (Kitzbichler et al., 2011). Notice that this interpretation of the results relies heavily on the idea of capacity limitations of each hemisphere, and assumes that the hemispheres can operate at least to some degree in parallel (Weissman et al., 2000), as well as a flexible and task-general role for frontal regions in performance on demanding cognitive tasks (Velanova et al., 2007; McGilchrist, 2010; Yeo et al., 2014). It remains a challenging task to be able to explain whether the benefits of cross-hemispheric communication reflect a predecision allocation of attentional resources (Banich, 1998), or a more online distribution of semantic or perceptual representations (Corbetta and Shulman, 2002; Stephan et al., 2007). The finding that visual attention benefits from a division of labor across the hemifields (Passarotti et al., 2002; Alvarez and Cavanagh, 2005) helps to substantiate the former conclusion.

In contrast, the finding of increasing segregation between word-specific frontotemporal regions and face-specific fusiform regions is in keeping with a rich body of knowledge describing cortical hemispheric specialization (Hervé et al., 2013), for both abstract language functions (Seghier and Price, 2011; Clarke et al., 2013) and fine-grained facial processing (Urgesi et al., 2005; Caldara and Seghier, 2009) in posterior temporal regions. For example, Seghier et al. (2011) demonstrated that this lateralization effect for words is inversely related to the effective connectivity in contralateral right hemisphere regions. Our results extend this idea to other domains of cognitive processing, and suggest a cognitive mechanism by which CFC is both regionally specific and parametrically modifiable.

An important theme in many studies of hemispheric dominance patterns focuses on whether the impulses traveling across the midline either promote or inhibit cross-hemispheric activity, and whether the callosum is generally supportive of hemispheric segregation or cooperation (Hellige et al., 1989; Chiarello and Maxfield, 1996). While our results may not directly differentiate these hypotheses - changes in BOLD-based connectivity could reflect excitatory or inhibitory changes in communication-our results, nonetheless, suggest a regional heterogeneity in the way different regions rely on cross-hemispheric communication. Our results suggest that cross-hemispheric mechanisms are dependent on not only the region examined but also the type of cognitive task performed. For example, a number of investigations of manual coordination have shown that callosally mediated interactions of bilateral motor cortex are a common correlate of fast, coordinated behaviors (Duque et al., 2005; Fling et al., 2011; 
Zilverstand et al., 2014), while a number of studies demonstrates a role for coordinated bilateral frontal activity in language comprehension (Yochim et al., 2005; Hartwigsen et al., 2010; Seghier et al., 2011). Future work may be able to tease apart these interpretations using more temporally sensitive measures.

\section{CFC mediates the relationship between DTI and BPA}

Last, our third finding was that for regions connected by the genu, CFC mediates the effect of genu FA on word-matching BPA, whereas for regions connected by the splenium, CFC in the splenium-occipital mediates the effect of splenium FA on facematching BPA. This finding fits well with our first finding regarding the role of genu FA in word-matching BPA and posteriorsplenium FA in face matching (Fig. 3). Furthermore, these findings demonstrate a strong a priori test of the relationship between connectivity measures (Honey et al., 2009), and suggest a role for neurocognitive modeling of structural-functional relationships that may compliment more data-focused approaches (Horn et al., 2014).

While an analysis of CFC demonstrated that frontopolar cortex CFC showed Difficulty-related increases for both word and face matching, face-matching BPA did not correlate with FA in the genu (Fig. 3A), which connects left and right frontopolar cortex, nor was any mediated relationship obvious. We interpret this apparent discrepancy in results as arising from differences in the adaptability of structural and functional connectivity. Whereas white-matter structure is static and may reflect individual differences in semantic-processing ability, the anterior frontal cortex can, nonetheless, be recruited bilaterally during the more difficult face-matching conditions to perform successfully on this cognitive task. This speculation would account for the presence of a functional, but not structural, correlate of bihemispheric interactions in face processing. Thus, component processes of perceptual and conceptual processing are attributable, at least in part, to callosal pathways, which may engender the integration of lateralized brain functions.

\section{Conclusions}

In sum, combining behavioral, white-matter, and functional measures, we investigated the where and when of crosshemispheric processing, focusing on the effects of difficulty and the BPA. Regarding where, we found that anterior and posterior callosal regions, respectively, mediate BPA for semantic word matching versus visual face matching. Regarding how, we found that when difficulty increases domain-general regions (frontopolar cortex) tend to collaborate, whereas domain-specific regions (temporopolar for word matching, fusiform for face matching) tend to segregate. Finally, linking where and how questions, we found that CFC mediates white-matter effects on crosshemispheric behavior.

\section{References}

Aboitiz F, Scheibel AB, Zaidel E (1992) Morphometry of the Sylvian fissure and the corpus callosum, with emphasis on sex differences. Brain 115: 1521-1541. CrossRef Medline

Alvarez GA, Cavanagh P (2005) Independent resources for attentional tracking in the left and right visual hemifields. Psychol Sci 16:637-643. CrossRef Medline

Atlas LY, Bolger N, Lindquist MA, Wager TD (2010) Brain mediators of predictive cue effects on perceived pain. J Neurosci 30:12964-12977. CrossRef Medline

Axmacher N, Schmitz DP, Wagner T, Elger CE, Fell J (2008) Interactions between medial temporal lobe, prefrontal cortex, and inferior temporal regions during visual working memory: a combined intracranial EEG and functional magnetic resonance imaging study. J Neurosci 28:7304-7312. CrossRef Medline

Banich MT (1998) The missing link: the role of interhemispheric interaction in attentional processing. Brain Cogn 36:128-157. CrossRef Medline

Banich MT, Belger A (1990) Interhemispheric interaction: how do the hemispheres divide and conquer a task? Cortex 26:77-94. CrossRef Medline

Baron RM, Kenny DA (1986) The moderator-mediator variable distinction in social psychological research: conceptual, strategic, and statistical considerations. J Pers Soc Psychol 51:1173-1182. CrossRef Medline

Berardi N, Bisti S, Maffei L (1987) The transfer of visual information across the corpus callosum: spatial and temporal properties in the cat. J Physiol 384:619-632. CrossRef Medline

Betzel RF, Byrge L, He Y, Goñi J, Zuo XN, Sporns O (2014) Changes in structural and functional connectivity among resting-state networks across the human lifespan. Neuroimage 102:345-357. CrossRef Medline

Binder JR, Desai RH, Graves WW, Conant LL (2009) Where is the semantic system? A critical review and meta-analysis of 120 functional neuroimaging studies. Cereb Cortex 19:2767-2796. CrossRef Medline

Butcher SJ, Cavanagh P (2008) A unilateral field advantage for detecting repeated elements. Percept Psychophys 70:714-724. CrossRef Medline

Caldara R, Seghier ML (2009) The Fusiform Face Area responds automatically to statistical regularities optimal for face categorization. Hum Brain Mapp 30:1615-1625. CrossRef Medline

Chiarello C, Maxfield L (1996) Varieties of interhemispheric inhibition, or how to keep a good hemisphere down. Brain Cogn 30:81-108. CrossRef Medline

Cisler JM, Bush K, Steele JS (2014) A comparison of statistical methods for detecting context-modulated functional connectivity in fMRI. Neuroimage 84:1042-1052. CrossRef Medline

Clarke A, Taylor KI, Devereux B, Randall B, Tyler LK (2013) From perception to conception: how meaningful objects are processed over time. Cerebral Cortex 23:187-197. CrossRef Medline

ClarkeS, MiklossyJ (1990) Occipital cortex in man: organization of callosal connections, related myelo- and cytoarchitecture, and putative boundaries of functional visual areas. J Comp Neurol 298:188-214. CrossRef Medline

Corbetta M, Shulman GL (2002) Control of goal-directed and stimulusdriven attention in the brain. Nat Rev Neurosci 3:201-215. Medline

Daselaar SM, Fleck MS, Prince SE, Cabeza R (2006) The medial temporal lobe distinguishes old from new independently of consciousness. J Neurosci 26:5835-5839. CrossRef Medline

Davis SW, Dennis NA, Buchler NG, White LE, Madden DJ, Cabeza R (2009) Assessing the effects of age on long white matter tracts using diffusion tensor tractography. Neuroimage 46:530-541. CrossRef Medline

Davis SW, Kragel JE, Madden DJ, Cabeza R (2012) The architecture of cross-hemispheric communication in the aging brain: linking behavior to functional and structural connectivity. Cereb Cortex 22:232-242. CrossRef Medline

Desikan RS, Ségonne F, Fischl B, Quinn BT, Dickerson BC, Blacker D, Buckner RL, Dale AM, Maguire RP, Hyman BT, Albert MS, Killiany RJ (2006) An automated labeling system for subdividing the human cerebral cortex on MRI scans into gyral based regions of interest. Neuroimage 31:968980. CrossRef Medline

Devereux BJ, Clarke A, Marouchos A, Tyler LK (2013) Representational similarity analysis reveals commonalities and differences in the semantic processing of words and objects. J Neurosci 33:18906-18916. CrossRef Medline

Duque J, Mazzocchio R, Dambrosia J, Murase N, Olivier E, Cohen LG (2005) Kinematically specific interhemispheric inhibition operating in the process of generation of a voluntary movement. Cereb Cortex 15:588-593. CrossRef Medline

Fillard P, Pennec X, Arsigny V, Ayache N (2007) Clinical DT-MRI estimation, smoothing, and fiber tracking with log-Euclidean metrics. IEEE Trans Med Imaging 26:1472-1482. CrossRef Medline

Fling BW, Walsh CM, Bangert AS, Reuter-Lorenz PA, Welsh RC, Seidler RD (2011) Differential callosal contributions to bimanual control in young and older adults. J Cogn Neurosci 23:2171-2185. CrossRef Medline

Han S, Weaver JA, Murray SO, Kang X, Yund EW, Woods DL (2002) Hemispheric asymmetry in global/local processing: effects of stimulus position and spatial frequency. Neuroimage 17:1290-1299. CrossRef Medline

Hartwigsen G, Price CJ, Baumgaertner A, Geiss G, Koehnke M, Ulmer S, Siebner HR (2010) The right posterior inferior frontal gyrus contributes to phonological word decisions in the healthy brain: evidence from dualsite TMS. Neuropsychologia 48:3155-3163. CrossRef Medline 
Hellige JB, Taylor AK, Eng TL (1989) Interhemispheric interaction when both hemispheres have access to the same stimulus information. J Exp Psychol Hum Percept Perform 15:711-722. CrossRef Medline

Hervé PY, Zago L, Petit L, Mazoyer B, Tzourio-Mazoyer N (2013) Revisiting human hemispheric specialization with neuroimaging. Trends Cogn Sci 17:69-80. CrossRef Medline

Honey CJ, Sporns O, Cammoun L, Gigandet X, Thiran JP, Meuli R, Hagmann P (2009) Predicting human resting-state functional connectivity from structural connectivity. Proc Natl Acad Sci U S A 106:2035-2040. CrossRef Medline

Horn A, Ostwald D, Reisert M, Blankenburg F (2014) The structuralfunctional connectome and the default mode network of the human brain. Neuroimage 102:142-151. CrossRef Medline

Ivry RB, Robertson LC (1998) The two sides of perception. Cambridge, MA: MIT.

Jones DK, Knösche TR, Turner R (2013) White matter integrity, fiber count, and other fallacies: the do's and don'ts of diffusion MRI. Neuroimage 73:239-254. CrossRef Medline

Kitzbichler MG, Henson RN, Smith ML, Nathan PJ, Bullmore ET (2011) Cognitive effort drives workspace configuration of human brain functional networks. J Neurosci 31:8259-8270. CrossRef Medline

Landis JR, Koch GG (1977) The measurement of observer agreement for categorical data. Biometrics 33:159-174. CrossRef Medline

Lavidor M, Walsh V (2004) The nature of foveal representation. Nat Rev Neurosci 5:729-735. CrossRef Medline

Linnet E, Roser ME (2012) Age-related differences in interhemispheric visuomotor integration measured by the redundant target effect. Psychol Aging 27:399-409. CrossRef Medline

Madden DJ, Spaniol J, Costello MC, Bucur B, White LE, Cabeza R, Davis SW, Dennis NA, Provenzale JM, Huettel SA (2009) Cerebral white matter integrity mediates adult age differences in cognitive performance. J Cogn Neurosci 21:289-302. CrossRef Medline

McGilchrist I (2010) Reciprocal organization of the cerebral hemispheres. Dialoques Clin Neurosci 12:503-515. Medline

Meyer AM, Federmeier KD (2008) The divided visual world paradigm: eye tracking reveals hemispheric asymmetries in lexical ambiguity resolution. Brain Res 1222:166-183. CrossRef Medline

Nelson DL, McEvoy CL, Schreiber TA (2004) The University of South Florida free association, rhyme, and word fragment norms. Behav Res Methods Instrum Comput 36:402-407. CrossRef Medline

Pandya DN, Seltzer B (1986) The topography of commissural fibers. In: Two hemispheres-one brain: functions of the corpus callosum (Lepore F, Ptito M, Jasper HH, eds), pp 47-74. New York: Alan R. Liss.

Park HJ, Kim JJ, Lee SK, Seok JH, Chun J, Kim DI, Lee JD (2008) Corpus callosal connection mapping using cortical gray matter parcellation and DT-MRI. Hum Brain Mapp 29:503-516. CrossRef Medline

Passarotti AM, Banich MT, Sood RK, Wang JM (2002) A generalized role of interhemispheric interaction under attentionally demanding conditions: evidence from the auditory and tactile modality. Neuropsychologia 40: 1082-1096. CrossRef Medline

Phillips PJ, Wechsler H, Huang J, Rauss PJ (1998) The FERET database and evaluation procedure for face-recognition algorithms. Image Vision Comput 16:295-306. CrossRef

Poffenberger AT (1912) Reaction time to retinal stimulation, with special reference to the time lost through nerve centers. Arch Psychol 23:1-73.

Rissman J, Gazzaley A, D’Esposito M (2004) Measuring functional connectivity during distinct stages of a cognitive task. Neuroimage 23:752-763. CrossRef Medline

Rodd JM, Davis MH, Johnsrude IS (2005) The neural mechanisms of speech comprehension: fMRI studies of semantic ambiguity. Cereb Cortex 15: 1261-1269. CrossRef Medline

Schulte T, Müller-Oehring EM, Rohlfing T, Pfefferbaum A, Sullivan EV (2010) White matter fiber degradation attenuates hemispheric asymme- try when integrating visuomotor information. J Neurosci 30:1216812178. CrossRef Medline

Schulte T, Maddah M, Müller-Oehring EM, Rohlfing T, Pfefferbaum A, Sullivan EV (2013) Fiber tract-driven topographical mapping (FTTM) reveals microstructural relevance for interhemispheric visuomotor function in the aging brain. Neuroimage 77:195-206. CrossRef Medline

Seghier ML, Price CJ (2011) Explaining left lateralization for words in the ventral occipitotemporal cortex. J Neurosci 31:14745-14753. CrossRef Medline

Seghier ML, Josse G, Leff AP, Price CJ (2011) Lateralization is predicted by reduced coupling from the left to right prefrontal cortex during semantic decisions on written words. Cereb Cortex 21:1519-1531. CrossRef Medline

Smith SM, Zhang Y, Jenkinson M, Chen J, Matthews PM, Federico A, De Stefano N (2002) Accurate, robust, and automated longitudinal and cross-sectional brain change analysis. Neuroimage 17:479-489. CrossRef Medline

Stephan KE, Marshall JC, Penny WD, Friston KJ, Fink GR (2007) Interhemispheric integration of visual processing during task-driven lateralization. J Neurosci 27:3512-3522. CrossRef Medline

Sullivan EV, Adalsteinsson E, Pfefferbaum A (2006) Selective age-related degradation of anterior callosal fiber bundles quantified in vivo with fiber tracking. Cereb Cortex 16:1030-1039. CrossRef Medline

Toussaint N, Soupet J-C, Fillard P (2007) MedINRIA: Medical Image Navigation and Research Tool by INRIA. In: Proceedings of MICCAI-07 Workshop on Interaction in Medical Image Analysis and Visualization. Brisbane, Australia.

Urgesi C, Bricolo E, Aglioti SM (2005) Hemispheric metacontrol and cerebral dominance in healthy individuals investigated by means of chimeric faces. Brain Res Cogn Brain Res 24:513-525. CrossRef Medline

van der Knaap LJ, van der Ham IJ (2011) How does the corpus callosum mediate interhemispheric transfer? A review. Behav Brain Res 223:211221. CrossRef Medline

Velanova K, Lustig C, Jacoby LL, Buckner RL (2007) Evidence for frontally mediated controlled processing differences in older adults. Cereb Cortex 17:1033-1046. CrossRef Medline

Wakana S, Caprihan A, Panzenboeck MM, Fallon JH, Perry M, Gollub RL, Hua K, Zhang J, Jiang H, Dubey P, Blitz A, van Zijl P, Mori S (2007) Reproducibility of quantitative tractography methods applied to cerebral white matter. Neuroimage 36:630-644. CrossRef Medline

Weissman DH, Banich MT, Puente EI (2000) An unbalanced distribution of inputs across the hemispheres facilitates interhemispheric interaction. J Int Neuropsychol Soc 6:313-321. CrossRef Medline

Worsley KJ, Friston KJ (1995) Analysis of fMRI time-series revisited-again. Neuroimage 2:173-181. CrossRef Medline

Xu D, Mori S, Solaiyappan M, van Zijl PC, Davatzikos C (2002) A framework for callosal fiber distribution analysis. Neuroimage 17:1131-1143. CrossRef Medline

Yarkoni T, Poldrack RA, Nichols TE, Van Essen DC, Wager TD (2011) Large-scale automated synthesis of human functional neuroimaging data. Nat Methods 8:665-670. CrossRef Medline

Yeo BT, Krienen FM, Eickhoff SB, Yaakub SN, Fox PT, Buckner RL, Asplund CL, Chee MW (2014) Functional specialization and flexibility in human association cortex. Cereb Cortex. Advance online publication. Retrieved April 25, 2015. doi: 10.1093/cercor/bhu217.

Yochim BP, Kender R, Abeare C, Gustafson A, Whitman RD (2005) Semantic activation within and across the cerebral hemispheres: what's left isn't right. Laterality 10:131-148. Medline

Zempleni MZ, Renken R, Hoeks JC, Hoogduin JM, Stowe LA (2007) Semantic ambiguity processing in sentence context: evidence from eventrelated fMRI. Neuroimage 34:1270-1279. CrossRef Medline

Zilverstand A, Sorger B, Zimmermann J, Kaas A, Goebel R (2014) Windowed correlation: a suitable tool for providing dynamic fMRI-based functional connectivity neurofeedback on task difficulty. PLoS One 9:e85929. CrossRef Medline 